\title{
La contribución de los programas de influencias psicosociales (complementados con otras estrategias) en el control de la epidemia tabáquica
}

\author{
José Precioso (*); Manuel Macedo (**) \\ (*) Instituto de Educação e Psicologia - Universidade do Minho, Braga, Portugal \\ $\left.{ }^{(*}\right)$ Hospital de San Marcos - Braga, Portugal
}

Enviar correspondencia a:

José Precioso. Instituto de Educação e Psicologia. Universidade do Minho. Campus de Gualtar, 4710-057, Braga, Portugal. E-mail: precioso@iep.uminho.pt.

\section{RESUMEN}

El mayor riesgo que tienen los niños y adolescentes cuando comienzan a fumar es el que se hagan dependientes del tabaco, muchas veces para toda su vida, como resultado del consumo diario de tabaco durante varios años, con lo que sufrirán algunas de las patologías causadas por el humo resultante de la combustión del tabaco, en particular de cáncer de pulmón, bronquitis crónica e infarto de miocardio. En este artículo se presentan algunas razones para prevenir el consumo de tabaco, se describe el proceso por el cual los niños y los jóvenes comienzan a fumar y la contribución que la escuela puede dar para evitar que inicien una carrera de fumadores perjudicial. De entre el conjunto de intervenciones preventivas a desarrollar en la escuela se recomienda la implantación de programas de influencias psicosociales y se describen algunos programas de este tipo. Para aumentar la eficacia de los programas de prevención en la escuela es necesario introducir modificaciones en el ambiente escolar, promover la implicación de los padres, desarrollar y aplicar programas de abandono para los adolescentes y adoptar estrategias de ámbito más comunitario como el control del acceso social y comercial al tabaco, campañas en los medios de comunicación, prohibición de la promoción y publicidad del tabaco y aumento de precios.

Palabras clave: prevención, tabaco, programas, influencia psicosocial.

\section{SUMMARY}

The main risk taken by children and adolescents commencing smoking is that, very often, they become tobacco dependent for their entire lives as a result of the daily use of tobacco over several years, leading to their suffering from the pathologies induced by inhaling tobacco smoke, in particular lung cancer, chronic bronchitis and myocardial infarction. This article presents some of the arguments for preventing smoking, describes the process through which youth starts smoking, and discusses the role that school could play in preventing students from becoming smokers. Among the range of preventive interventions to be made in schools, the introduction of psychosocial influence programmes is recommended with a description of some of the programmes of this kind. In order to increase the effectiveness of prevention programmes in schools, it is necessary to introduce modifications in the school environment, promote the involvement of parents, develop and apply teen cessation programmes and adopt more community-based approaches such as controlling social and commercial access to cigarettes, media campaigns, banning cigarette advertising and increasing prices.

Key words: prevention, cigarettes, tobacco, programme, psycho-social.

\section{INTRODUCCIÓN}

$E$ consumo de tabaco durante la infancia y la adolescencia es un serio problema, presente y futuro, de salud pública. Los niños y los jóvenes que fuman, activa o pasivamente, ven aumentado el riesgo de padecer amigdalitis, catarrros, bronquitis, ataques de asma, así como de otros problemas respiratorios (Melero, Flores y Anda, 1997a); National Clearinghouse on Tobacco and Health, 1993; Ribeiro, 1988; U.S.D.H.H.S., 1994; 1997). Sin embargo la severidad de los efectos a corto plazo del consumo de tabaco fumado en la salud, el mayor riesgo que corren los niños y adolescentes cuando comienzan a fumar es el 
de hacerse dependientes del tabaco, muchas veces para toda su vida, pudiendo mas tarde llegar a padecer alguna de las graves patologías asociadas a la inhalación del humo del tabaco. Se estima que entre el 70 y el $90 \%$ de las personas que consumen cuatro o más cigarrillos por día durante algún tiempo pueden convertirse en fumadores dependientes (Becoña y Vázquez, 1996; Vries y Kok, 1986). Estudios mas recientes efectuados por DiFranza et al. (2002), muestran que los niños se hacen dependientes de la nicotina más fácilmente que los adultos y con cantidades de tabaco tan bajas que nunca antes habían sido estudiadas (fumar dos cigarrillos por día durante cuatro a seis semanas). Los investigadores constataron además que las chicas se hacían más fácilmente dependientes de la nicotina que los chicos.

Varios estudios epidemiológicos muestran que la mayoría de los actuales fumadores comenzaron a fumar antes de los 18 años de edad y que la probabilidad de llegar a ser fumador regular es tanto mayor cuanto menor es la edad en que se comienza a fumar (Becoña y Vázquez, 1996). Varios autores constataron también que el consumo de tabaco puede aumentar el riesgo de dependencia de otras sustancias (Becoña y Vázquez, 1996; Gold, 1996; Indiana Prevention Resource Center, 1998).

El conjunto de los datos que referimos anteriormente muestran la importancia de prevenir el consumo de tabaco en los jovenes, para controlar la expansión de la epidemia tabáquica y, consecuentemente, evitar los problemas de salud, económicos, sociales y ambientales a ella asociados. En las siguientes páginas de este artículo intentaremos clarificar el proceso por el cual los niños y los jóvenes comienzan a fumar, demostrar que la prevención puede tener un papel importante en la reducción de la prevalencia del consumo y la contribución que la escuela puede hacer para prevenir la expansión de la epidemia tabáquica.

\section{Síntesis del proceso por el cual los jóvenes se hacen fumadores: la "carrera del fumador"}

Para intentar prevenir el consumo de cualquier droga es necesario comprender su etiología. En el caso concreto del tabaco, cuándo y por qué se comienza a fumar. Se admite que en los países desarrollados la mayoría de los fumadores comienzan a fumar en la adolescencia, antes de los 18 años de edad. Sin embargo, en Portugal, un porcentaje elevado de fumadores, cerca del 30\%, comienzan a fumar más tarde (Brandão, 2003; Nunes, 2002; Precioso, 2003), lo que hace indispensable actuar al nivel de la prevención en la Universidad y en el lugar de trabajo. Después de investigaciones sistemáticas, se admite que no existe una causa única que explique esta conducta. Parece haber un conjunto de factores complejos e interrelacionados que predisponen a los mas jóvenes a fumar (Pestana y Mendes, 1999; Ulster Cancer Foundation, 1988). Por otro lado, se conoce que los factores que llevan a las personas a comenzar a fumar difieren de individuo a individuo, lo que hace que los esfuerzos preventivos sean complejos pues se trata de actuar sobre una realidad heterogénea. No obstante la complejidad de esta conducta, existen algunas teorías que pretenden explicar por qué los jóvenes comienzan a fumar y que pueden tener un importante valor en la orientación de los esfuerzos preventivos.

En opinión de varios especialistas (Bellew y Wayne, 1991; Flay, 1985; Hill, 1991; Nutbeam, Mendoza y Newman, 1988), la adquisición de cualquier hábito, y fumar es apenas un ejemplo, no es una simple respuesta a un estímulo. Es un proceso que probablemente se desarrolla a lo largo del tiempo, siguiendo una secuencia ordenada de etapas. Parece consensuado que existen básicamente de cuatro a cinco etapas hasta que el niño se convierte en fumador dependiente: la preparación, la prueba, la experimentación, la habituación (consumo regular) y el mantenimiento (consumo dependiente).

Según el U.S.D.H.H.S. (1994) y Becoña y Vázquez (1998), en la fase de preparación el niño puede comenzar a formar actitudes y creencias positivas sobre fumar y los principales factores de riesgo asociados a esta fase son los modelos de los adultos y de la familia (principalmente los padres y hermanos).

Después de la prueba del primer cigarrillo sigue la fase de experimentación, o sea la del consumo esporádico de cigarrillos. Para el paso de esta fase es particularmente determinante la influencia activa o pasiva de los amigos, la percepción de que fumar es normal y la disponibilidad de cigarrillos.

En la fase de habituación el adolescente comienza a fumar con alguna regularidad (por lo menos un cigarrillo por semana) y los principales factores psicosociales de riesgo asociados a este tipo de consumo son: tener amigos fumadores, estar implicado en situaciones sociales en las que los amigos apoyan el consumo, tener baja auto-eficacia y capacidad de rechazo, tener disponibilidad de cigarrillos, percibir que fumar es útil para la persona, y tener pocas restricciones a fumar en la escuela y en la comunidad.

En la fase de mantenimiento/dependencia el indivíduo continúa fumando debido a la dependencia física de la nicotina y a la dependencia psicológica y social (Becoña y Vázquez, 1998; U.S.D.H.H.S., 1994).

Otra teoria que procura explicar el consumo de tabaco es la Teoría de la Acción Razonada (Ajzen y Fishbein, 1980). Según esta teoria, el comportamiento de fumar puede ser predicho por la intención de la persona a fumar, aunque la realización de esa intención pueda no ser posible debido a ciertas barreras (por 
ejemplo, no se quiere fumar pero no sabe como rechazar un cigarrillo; quiere fumar pero no tiene dinero para comprar cigarrillos) (Vries y Kok, 1986; Vries, 1989).

Según la Teoría de la Acción Razonada la intención está "determinada" por la actitud y la norma subjetiva. La actitud representa el sentimiento general (favorable o desfavorable) relativo a un comportamiento (por ejemplo, fumar es malo) (Lima, 1993). La actitud de la persona relativa a un comportamiento está influenciada por sus creencias sobre las consecuencias (sobre los costes y beneficios, o sea, las ventajas y desventajas) de la realización del mismo. La norma subjetiva de una persona corresponde a la percepción personal sobre si la mayoría de las personas que son importantes para ella piensan que la persona debe o no realizar un determinado comportamiento (Vries, 1989). En opinión de Cruz y Vilaça (1996), mide el grado de presión social percibido por la persona para realizar el comportamiento.

En resumen, de acuerdo con esta teoría las personas tienen mayor probabilidad de realizar un determinado comportamiento cuando tienen una actitud favorable cara a su realización y cuando perciben apoyo normativo para su realización por parte de personas que ellas consideran importantes o que les merecen credibilidad.

La validez de este modelo para explicar la conducta de fumar fue investigada entre otros autores por Vries y Kok (1986) y Vries (1989). Estos autores verificaron que los niños fumadores tienen de hecho una actitud positiva en relación a fumar, o sea, consideran que fumar es bueno y agradable, en cuanto los no fumadores tienen una actitud negativa y ven fumar como teniendo los efectos opuestos. Los estudios realizados por Vries, Dijkstra y Kuhlman, (1988) muestran también que los niños no fumadores perciben una mayor norma subjetiva negativa en lo que respecta a fumar regularmente, debida a la influencia de sus padres, hermanos, parientes, médicos, no fumadores y amigos no fumadores.

Esta teoría y las investigaciones realizadas para validarla tiene fuertes implicaciones en el diseño de programas de prevención del consumo de tabaco. Se supone que la promoción de actitudes favorables a no fumar y el desarrollo de una norma subjetiva desfavorable al consumo de tabaco pueden tener un elevado efecto preventivo.

En opinión de Vries y Kok (1986) y Vries (1989), para influenciar las actitudes, un programa de prevención debe: 1. Destacar las ventajas de no fumar y las desventajas de fumar. 2. Proporcionar información sobre los efectos a corto plazo pues los niños generalmente consideran las consecuencias a largo prazo como distantes en el tiempo y por ello poco probables de que les afecten. 3. Proporcionar argumentos para contradecir la tendencia que los fumadores tienen de minimizar las desventajas de fumar. 4. Proteger a los no fumadores de las presiones para fumar, demostrando las imprecisiones de algunas ventajas percibidas por los fumadores (por ejemplo, que es relajante) e indicando que las supuestas ventajas de fumar referidas (a veces percibidas por los fumadores) pueden ser realizadas por otras actividades alternativas (por ejemplo, bailar, conversar y practicar un deporte). 5. Entrenar a los no fumadores para contradecir los (falsos) argumentos de los fumadores, proporcionándoles contra-argumentos y desarrollando su asertividad.

Vries y Kok (1986) y Vries (1989), proponen igualmente algunos métodos que pueden emplearse para influenciar las normas subjectivas de los no fumadores. En su opinión, para influenciar la norma subjectiva, el programa de prevención debe: 1. Explicitar que la mayoría de las personas no fuman y están en contra de que fumen otras personas. 2. Demostrar los procesos directos y de modelado por los cuales los fumadores influencian a los no fumadores. 3. Aumentar la resistencia en los no fumadores a las presiones ejercidas por los fumadores. 4. Discutir los peligros de las situaciones de desafio en las cuales las presiones para fumar son más fuertes. 5. Desarrollar competencias en los no fumadores para afrontar las presiones para fumar en situaciones de desafío. 6. Aumentar las oportunidades de mantener el comportamento de no fumar empleando técnicas de compromiso tales como la presentación pública de la decisión de no fumar (frente a una audiencia) o redactar y firmar un contrato de no fumar.

\section{Los programas de influencias psicosociales en la prevención del tabaquismo}

Los modelos que mejor explican la conducta de fumar son los bio-psico-socio-ambientales, o sea, los que refieren que los comportamientos están determinados por factores biológicos, psicológicos, sociales y ambientales y que para poder modificar o alterar esa conduta tendremos que actuar a través de programas que pretendan actuar sobre esos factores. Hoy se defiende que la prevención del consumo de tabaco debe ser hecha de forma global y comprensiva, dirigida a los distintos factores de riesgo asociados al consumo. Está demostrado que los programas preventivos de influencia psicosocial aplicados en la escuela a los alumnos tienen demostrado su elevada eficacia preventiva y deben por eso formar parte de esa estrategia global de prevención.

A través de la elaboración y aplicación de los programas de influencias psicosociales se intenta "inmunizar" a los niños y jóvenes contra el tabaquismo. Tal como el organismo desarrolla inmunidad contra un agente infeccioso al que se le ha sometido en dosis 
que no le hagan enfermar, también podemos desarrollar en el alumno una cierta "inmunidad" contra las influencias psicosociales que lo pueden llevar a fumar. A través de los programas intensivos de influencias psicosociales inspirados en las teorias descritas anteriormente podemos desarrolar en el alumno resistencias psicológicas contra algunos factores psicosociales que conducen al consumo de tabaco, concretamente, promover el desarrollo de una actitud negativa en relación a fumar y aumentar su auto-eficacia que es una cualidad determinante para que resista las presiones de los iguales para que fume. Dado que muchos niños comienzan a fumar alrededor de los 12-15 años, el proceso de "vacunación" debe comenzar antes de esa edad, o sea en el $6^{\circ}$ y $7^{\circ}$ año de la escolaridad, y continuar con la aplicación de sesiones de refuerzo, especialmente en la transición del ciclo de enseñanza (del básico para el secundario y de éste para la universidad).

\section{Breve caracterización de algunos programas de influencias psicosociales}

\author{
El programa "No fumar es lo que va a pasar" ("Não
} fumar é o que está a dar")

El programa de prevención del consumo de tabaco "No fumar es lo que va a pasar" ("Não fumar é o que está a dar") es un programa intensivo, específico y transversal, de prevención del consumo de tabaco, de influencias psicosociales, constituido por un conjunto de 15 sesiones semanales de una hora de duración, dirigidas a los alumnos del $7^{\circ}$ año de escolaridad portuguesa (12 años) (que son los alumnos en mayor riesgo de comenzar a fumar), a ser aplicadas en las disciplinas de Ciencias Naturales, Lengua Portuguesa, Educación Visual, Matemáticas y Educación Física.

Las sesiones del programa "No fumar es lo que va a pasar" están agrupadas en seis componentes básicos: información sobre el humo del consumo de tabaco; construir una actitud hacia fumar; tomar una decisión sobre el futuro uso del tabaco; corregir la percepción exagerada del número de amigos fumadores; $y$, resistir las influencias sociales para fumar.

Un componente corresponde a una sesión o a un conjunto de sesiones dirigidas a contradecir un factor determinado del riesgo asociado al comienzo de fumar por los jóvenes.

El currículo del programa "No fumar es lo que va a pasar" y su contenido se encuentra en http//: www.iep.uminho.pt/home/precioso. Este programa también está publicado (Precioso, 2000).

El programa que le sirve de refuerzo, denominado "Aprende a cuidar de ti", está destinado a los alumnos del $8^{\circ}$ año y se debe aplicar en el año siguiente en las disciplinas de Ciencias Naturales, Portugués y Matemáticas (Precioso, 2001). Como ya se ha indicado, además de constituir un refuerzo del programa principal, pretende simultaneamente desarrollar en el alumno la intención de optar por un estilo de vida

\section{El programa "Querer es poder"}

El programa "Querer es poder", está constituido por 6 sesiones y se dirige a los jóvenes de 12 a 14 años, desarrollado en Portugal en el ámbito del European Smoking Prevention Framework Approach (ESFA, 1998, a, b).

Como otros programas de tipo psicosocial posee, entre otros, cuatro componentes básicos: un componente informativo que proporciona a los alumnos la comprensión de las consecuencias negativas que fumar tiene en la salud; un componente que ayuda a los alumnos a identificar la procedencia de las influencias para fumar; un componente de entrenamiento en habilidades sociales para resistir las presiones de los compañeros para fumar; y, un componente en el que los alumnos se comprometen públicamente a no fumar y a exponer las razones que los llevan a tomar esa decisión.

Información sobre este programa puede obtenerse a través del Conselho de Prevenção do Tabagismo, en Lisboa.

\section{El programa "¡No Gracias, no fumo!".}

El programa consta de seis sesiones a aplicar con una frecuencia semanal.

El programa tiene también previstas cuatro lecciones de refuerzo a aplicar en los dos años siguientes después de finalizar el programa. Dos sesiones deben aplicarse, en dos semanas consecutivas, después de un año y otras dos también con una frecuencia semanal, pasados dos años de la aplicación del programa. Según los autores del programa éste debe ser aplicado a los alumnos de 11-12 años (Becoña, Palomares y García, 1994).

Tal como ocurre en otros programas de influencias psicosociales ya descritos, el programa " ¡No gracias, no fumo!" tiene, aparte de otros, tres componentes considerados fundamentales: información, entrenamiento en habilidades y toma de decisiones. En las sesiones relacionadas con el componente de información se utilizan métodos de aprendizaje activo. En vez de ser el profesor el que proporciona la información, se le pide a los alumnos que, divididos por grupos, la consigan y la traigan para el aula. A cada grupo se les da un tema para tratar. La información que se pretende que los alumnos recojan y traigan para el aula se refiere fundamentalmente a las consecuencias a corto plazo del consumo de tabaco, tanto de carácter fisiológico como social, por ser éstas las de mayor relevancia 
para los adolescentes. Según Becoña, Palomares y García, (1994) y Becoña (S/D), el segundo componente es probablemente el más importante del programa y consiste en el entrenamiento en habilidades que permitan a los adolescentes rechazar los ofrecimientos de cigarrillos que eventualmente puedan hacerles otros jóvenes de su edad, sin perder su amistad y sin sentirse marginados por el grupo. El entrenamiento de estas habilidades se realiza por medio de representaciones en la clase, en las cuales se simulan situaciones relacionadas con la oferta de cigarrillos que los propios alumnos identificaron como siendo las que con mayor probabilidad les pueden ocurrir en la realidad.

Como ya fue indicado, de acuerdo con la teoría de la inoculación social, los sujetos que hayan sido expuestos a situaciones controladas, en las cuales tienen que defender una determinada postura, tienen mayor probabilidad de mantenerla luego en una situación real.

El tercer componente del programa consiste en una toma de decisiones sobre la futura intención relativa al consumo de tabaco y un compromiso público relativo a la decisión tomada. En primer lugar, se le pide a cada uno de los alumnos que defina las razones por las cuales decidió que será un no fumador en el futuro y después que exponga su propia decisión y las razones que le llevaron a ella delante de sus compañeros de clase. Con este ejercicio, además de clarificar su postura, cada alumno se compromete con ella y se somete a su futura presión de disonancia cognitiva, o sea, difícilmente hará una cosa contraria a aquella con la que se comprometió delante de sí mismo y de otros.

Conforme ya indicamos, el programa cuenta con algunas sesiones de refuerzo. La función de las sesiones de recuerdo es mantener el efecto del programa en los alumnos a quienes fue aplicado. Estas sesiones constituyen fundamentalmente una recapituación de las nociones proporcionadas durante la implementación del programa (como las limitaciones impuestas por el tiempo).

En la primera sesión de recapitulación a efectuar en el primer año después de la aplicación del programa son abordadas las consecuencias negativas de fumar cigarrillos, los motivos que llevan a los jóvenes a iniciarse en el consumo y los medios utilizados por la publicidad para inducirlos a consumir. También se realizan entrenamientos de cómo rechazar ofrecimientos de cigarrillos sin ser rechazados y sin ofender a aquel, o aquellos, que se lo ofrecen. Por último, se le pide a los alumnos que construyan un póster antitabaco, que puede ser utilizado al año siguiente como ejemplo para los cursos en los que el programa se aplica por primera vez o para reproducir en camisetas, llaveros, pins, etc., que los autores sugieren que pueden ser vendidos por los propios alumnos para recoger fondos para su viaje de fin de curso.
La segunda sesión de recuerdo, también a llevarse a cabo durante el primer año después de la aplicación del programa, incluye la estimación del número de fumadores y la corrección de la percepción equivocada del porcentaje de fumadores que los alumnos eventualmente puedan tener. También se intenta en esta lección modificar la percepción positiva que los alumnos puedan haber creado relativa a la desaparición de los síntomas que ocurren cuando se fuma las primeras veces. En esta segunda sesión de recapitulación se realiza de nuevo la expresión pública de los motivos que los alumnos tienen para no fumar y de las futuras intenciones relativas al consumo de tabaco. Finalmente, se realiza la elección por toda la clase del mejor cartel realizado.

En la tercera sesión de recuerdo (primera del segundo año después de la aplicación del programa) son de nuevo abordados los motivos a corto plazo para no fumar; se practica de nuevo el rechazo de oferta de cigarrillos, a través de representaciones en grupo, de forma que sean lo más semejantes posibles a lo que ocurrirá en la realidad; se analizan de nuevo los métodos utilizados por los fabricantes de cigarrillos para inducirlos a fumar y se les pide a los alumnos que confeccionen un póster antitabaco usando los métodos que ellos mismos observaron que son los utilizados en los anuncios de cigarrillos. Esta actividad tiene como finalidad hacer que los alumnos desmonten las estrategias utilizadas por la publicidad y utilicen las mismas "armas" para combatir los efectos de esa misma publicidad.

En la sesión número cuatro de recuerdo se vuelve a comprobar y a modificar (si fuera necesario; esto es, si los alumnos aún sobreestiman el porcentaje de fumadores) la percepción normativa del hábito de fumar, solicitándose de nuevo una declaración de intenciones y un compromiso público sobre fumar o no fumar, presentándose información sobre el papel del tabaco para el paso al consumo de otras drogas y se realiza una elección del mejor trabajo antitabaco de la clase.

La aplicación del programa puede ser realizado por aplicadores de fuera de la escuela o por profesores entrenados especialmente para ello en una sesión de tres horas de duración.

El papel de cualquier de estos dos tipos de aplicadores, ayudados por los líderes elegidos por los alumnos, es dirigir las discusiones y las actividades a realizar.

La evaluación de la eficacia del programa se efectuó tanto en la población escolar de Arteixo (A Coruña), con un diseño experimental de grupo experimentalgrupo control, como en otro estudio más amplio en distintas escuelas con la utilización de un grupo experimental y un grupo de control, demostrándose en ambos casos la eficacia del programa. 
Los programas de influencias psicosociales poseen elevada eficacia en la prevención del consumo de tabaco

Un estudio cuasi-experimental realizado entre 19972000 indicó que el programa "No fumar es lo que va a pasar" consiguió alcanzar resultados altamente positivos (por lo menos a corto plazo), en lo que respecta al control de algunos factores de riesgo relacionados con el comienzo del consumo de tabaco y la prevención del consumo de tabaco. Se constató la mejora del nivel de conocimientos de los alumnos; disminución de la percepción por parte de los alumnos de que a sus amigos les gustaría que ellos fumasen, probablemente porque consiguió persuadir a los alumnos de que no los presionasen sus amigos para fumar; mejorar su capacidad de decir no; fortalecer el sentimiento de vulnerabilidad de los alumnos y su preocupación con las consecuencias de fumar; incrementó una actitud desfavorable relativa al consumo de tabaco y redujo la percepción exagerada del porcentaje de iguales fumadores (sobreestimación). El programa tiene un efecto positivo en prevenir el consumo total de tabaco. No obstante la nota más destacable es el hecho de que el programa ha conseguido resultados apreciables en prevenir el consumo diario de tabaco.

En relación al consumo total de tabaco la intervención tiene un efecto semejante al alcanzado por otros programas (ej., Sanz, 1993, Sanz y Gándara, 1997). En lo que se refiere al consumo diario de tabaco se obtienen resultados a corto plazo muy próximos al programa diseñado e implementado por el Norwegian Cancer Society y del Research Center for Health Promotion University of Bergen (Josendal, Aaro y Bergh, 1998).

Los trabajos de revisión efectuados por muchos autores, como Best et al. (1988), Llanbrich y Villalbi (1990) y Becoña et al. (1994), indican que los programas más eficaces en la prevención del abuso de sustancias implementados en la escuela son los que refuerzan la capacidad personal de resistir las presiones sociales. Diversos programas basados en este modelo, con una duración que oscila entre ocho y diez horas en un año, aplicados entre el sexto y el octavo año, conseguirán reducciones significativas en el inicio del tabaquismo. Otros programas basados en el refuerzo de la autoestima o de prevención inespecífica no mostraron resultados positivos apreciables al aplicarse a estas edades y en algunos casos hasta fueron contraproducentes (Llanbrich y Villalbi, 1990)

La revisión de la eficacia de algunos programas mostró que las intervenciones que presentaban buenos resultados incluían los siguientes componentes: se basaban en un aprendizaje de tipo socrático, bidi- reccional e interactivo (le llevan al alumno a descubrir las cosas por medios propios); insisten en las consecuencias negativas de fumar ya conocidas por los alumnos (sobre todo a corto plazo) en vez de centrarse en aumentar los conocimientos sobre las mismas (se profundiza mucho en los conocimientos sobre una determinada desventaja de fumar); se desarrollan habilidades para resistir las presioness sociales; se elimina la percepción errada de que el consumo es un comportamiento normal de conducta social; se suscitan declaraciones públicas de rechazo del consumo por parte de los alumnos.

Gracias a varios estudios experimentales y cuasiexperimentales, llevados a cabo tanto por nosotros como por otros autores, y también en base a la revisión de varias investigaciones realizadas, podemos afirmar que las intervenciones preventivas basadas en modelos teóricos adecuados, bien planificadas y bien implantadas, pueden reducir la tasa del consumo de tabaco (y de otras drogas) y lógicamente los problemas a él asociados. Estamos, por tanto, completamente de acuerdo con Becoña (1999), cuando afirma que no aplicar programas preventivos eficaces que consideren los factores de riesgo y protección del consumo, hará que el problema del consumo de drogas legales e ilegales disminuya menos que lo deseable, se mantenga o incluso hasta siga aumentando.

Dada la demostrada eficacia de los programas preventivos y el reducido coste de los mismos en lo que podríamos llamar la "primera dosis de la vacuna de la prevención contra el consumo de tabaco", pensamos que la aplicación de los programas debe generalizarse a todas las escuelas.

Su difusión en las escuelas, podría ser hecha de acuerdo con las cuatro fases sugeridas por Becoña (1995) para la difusión de los programas de prevención. En la fase de diseminación, el programa deberá ser divulgado y las escuelas persuadidas de que lo integren en su proyecto educativo, más concretamente, en su plan de actividades. En la fase de adopción, deberán realizarse iniciativas para que las escuelas se comprometan en la implementación del programa. En la fase de implementación deberá organizarse la formación para que los profesores puedan implementar el programa sin sobresaltos. En la fase de mantenimiento, y una vez que el programa haya sido adoptado por las escuelas, deberán llevarse a cabo las acciones necesarias para garantizar que las escuelas mantengan el programa en su plan de actividades.

La difusión de estos programas necesita del apoyo de las Autoridades de Salud y de Educación de nuestro país. 
Otras iniciativas a desarrollar en la escuela para aumentar la eficacia de la prevención tabáquica

La aplicación de programas preventivos de influencias psicosociales debe integrar una estrategia más comprensiva de la prevención del consumo de tabaco, que procuramos esquematizar en la figura 1, y que está de acuerdo con las ideas de Perry y Forster (2002).

Entre las iniciativas y actividades preventivas en el consumo de tabaco implementadas en el medio escolar dirigidas directamente a los alumnos se sugieren: la aplicación generalizada de programas de prevención intensivos del tipo de influencias psicosociales (en Portugal tenemos el programa "No fumar es lo que va a pasar" y el programa "Querer es Poder"; acciones extracurriculares, como por ejemplo la creación de clases sin fumadores (Nebot, 1999; Savolainen, 1998; Wiborg y Hanewinkel, 1999); el desarrollo y aplicación de programas de deshabituación para jóvenes; la continuidad de la intervención antitabáquica a lo largo del curso escolar (Becoña, 1995; 1999; Centers for Disease Control and Prevention, 1994); la promoción de estilos de vida saludables, a través del desarrollo de un curriculo transversal de Educación para la Salud (Mendoza, 1999; Nebot, 1999). Entre las iniciativas y actividades preventivas a implementar en el medio escolar que pueden dirigirse indirectamente a los alumnos se sugieren la creación de un ambiente escolar (escuela sin tabaco) que refuerce las acciones educativas ejercidas directamente sobre los alumnos (ESFA, 1998b; ESFILES, 1999).

Entre las iniciativas y actividades preventivas implementadas en la comunidad que pueden dirigirse indirectamente a los alumnos se sugieren: el refuerzo de las acciones educativas sobre las desventajas del fumador activo y pasivo dirigidas a los adultos, especialmente a los padres de alumnos (Centers for Disease Control and Prevention, 1994); la implicación de las familias en la prevención del consumo de tabaco por parte de los hijos, a través de sesiones en las escuelas, envío de folletos, a través del contacto con el jefe de clase; la elaboración y disponibilidad de programas de deshabituación para adultos (Centers for Disease Control and Prevention, 1994); Nebot, 1999); la monitorización y el refuerzo de la inversión en la prevención primaria y la creación de espacios de ocio alternativos (espacios verdes y equipamientos que aparten a los jóvenes de locales de mayor riesgo de poder fumar, como son cafés, bares, salas de juego, etc.).

\section{FIGURA 1}

Prevención del consumo de tabaco por los jóvenes

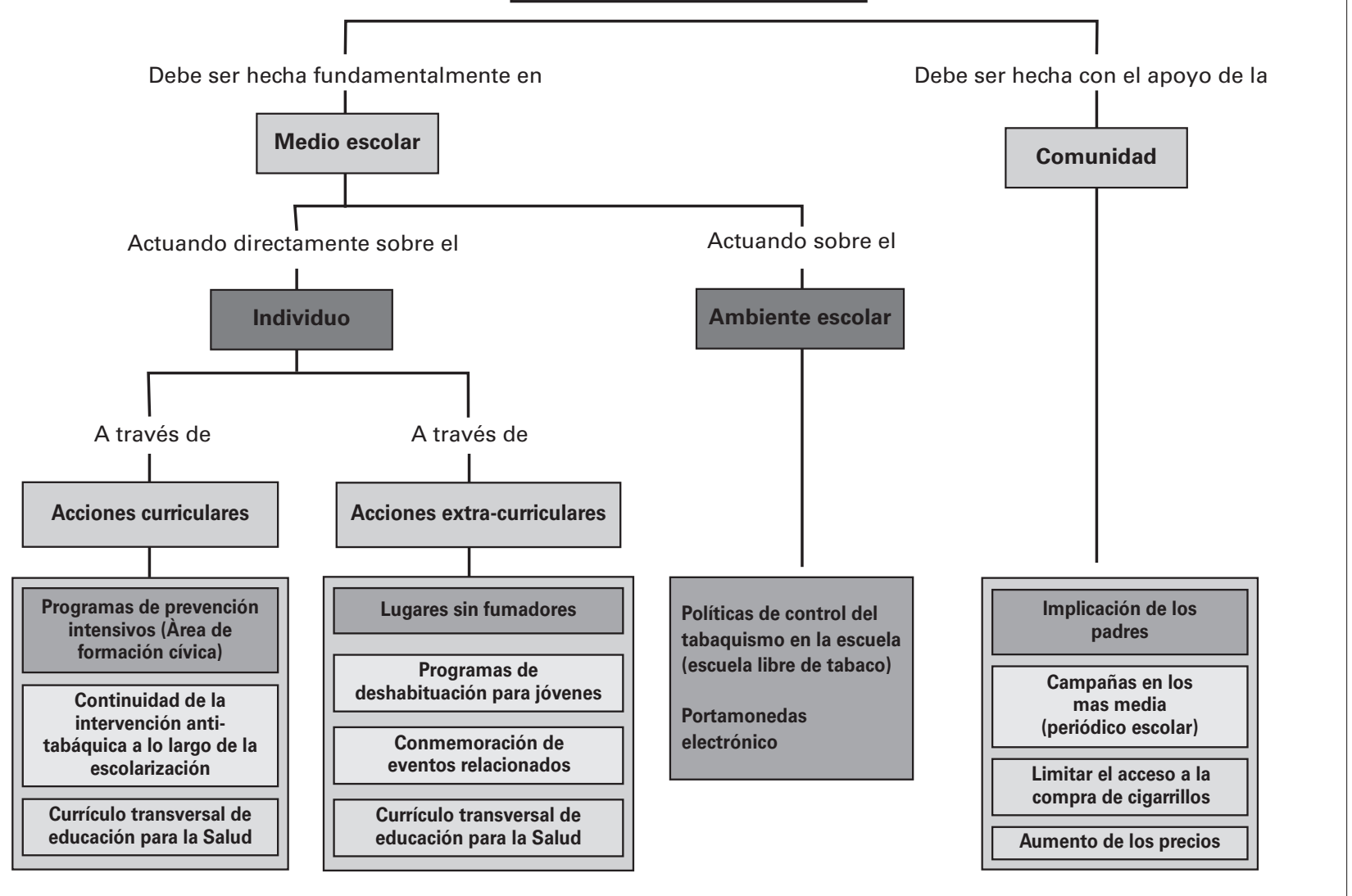




\section{REFERENCIAS BIBLIOGRÁFICAS}

Ajzen, I. y Fishbein, M. (1980). Understanding attitude and predicting social behavior. Englewood Cliffs, NJ: Prentice Hall.

Becoña, E. (S/D). No gracias no fumo. Santiago de Compostela: Universidade de Santiago de Compostela (documento no publicado).

Becoña, E. (1995). La prevención de las drogodependencias. Introducción a algunas cuestiones actuales. En Becoña, E., Rodríguez, A. y Salazar, I. (1995). Drogodependencias IV. Prevención. Santiago de Compostela: Servicio de Publicaciones e Intercambio Científico de la Universidad de Santiago de Compostela.

Becoña, E. (1999). A prevenção de drogodependências em adolescentes. En Precioso, J., Viseu, F., Dourado, L., Vilaça, T, Henriques, R y Lacerda, T. (1999). Educação para a Saúde. Braga: Departamento de Metodologias da Educação. Universidade do Minho.

Becoña, E., Palomares, A. y García, M.P. (1994). Tabaco y Salud: Guia de prevención y tratamiento del tabaquismo. Madrid: Ediciones Pirámide.

Becoña, E. y Vázquez, F. (1996). La Prevención del Tabaquismo: una necesidad para la mejora de la salud y del bienestar. Extensiones, 3(1 e 2), 21-29.

Becoña, E. y Vázquez, F. (1998). Tratamiento del tabaquismo. Madrid: Dykinson.

Bellew, B. y Wayne, D. (1991). Prevention of smoking among schoolchildren: a review of research and recommendations. Health Education Journal, 50 (1), 3-7.

Best, J., Thomson, S., Santi, S., Smith, E. e Brown, K. (1988). Preventing Cigarette smoking among school children. Annual Review of Public Health, 9, 161-201.

Brandão, $\mathrm{M}^{\mathrm{a}}$ (2002). Atitudes, conhecimentos e hábitos tabágicos dos professores dos $2^{\circ}$ e $3^{\circ}$ ciclos do ensino básico do Porto. Faculdade de Medicina e Instituto de Ciências Biomédicas Abel Salazar - Universidade do Porto.

Centers for Disease Control and Prevention (1994). Guidelines for School Health Programs to Preventing Tobacco Use and Addiction. U.S. Department Health and Human Service. MMWR; 43(rr-2), 1-20.

Cruz, J. y Vilaça. Ma (1996). Prevenção do SIDA nos adolescentes e jovens adultos: contribuições da Psicologia. Psicologia: Teoria, Investigação e Prática, 1, 219-242.

DiFranza, J, Savageau, J., Rigotti, N., Flectcher, K., Ockene, J., MacNeil, A., Coleman, M. y Wood (2002). Development of symptoms of tobacco dependente in youths: 30 month follow up data from the DANDY study. Tobacco Control, 11, 228-235.

ESFA (1998). Querer é poder: programa de prevenção do tabagismo para jovens dos 12 aos 14 anos. Conselho de Prevenção do Tabagismo (documento no publicado).

ESFA (1998). Towards a smoke-free school: a step-by-step guide on how to create a smoke-free school. Maastricht (documento no publicado).
ESFILES (1998). Towards a smoke-free school: a step-bystep guide on how to create a smoke-free school. Maastricht (documento no publicado).

Flay, B. (1985). Psychosocial approaches to smoking prevention: a review of findings. Health Psychology, 4 (5) 449488.

Gold, M. (1996). Tabaco. Barcelona: Ediciones en Neurociencias.

Hill, D. (1991). Causes of smoking in children. World Conference on Tobacco and Health-The Global War-Proceedings of the Seventh World Conference on Tobacco and Health, Perth Western Australia, 205-209.

Indiana Prevention Resource Center (1998). Factline on tobacco. Indiana University.http: //www.drugs.indiana.edu/publications/iprc/factline/tobac.html.

Josendal, O., Aaro, L. y Bergh, I. (1998). Effects of a schoolbased smoking prevention program among subgroups of adolescents. Health Education Research, 13(2), 215224.

Llanbrich, J. y Villalbí, J. (1990). Prevención desde la escuela del uso de tabaco y de otras substancias adictivas. Gaceta Sanitaria, 4 (17), 70-75.

Lima, M. (1993). Atitudes. En Vala, J. e Monteiro, M. (1993). Psicologia Social. Lisboa: Serviço de Educação da Fundação Caloust Gulbenkian.

Melero, J., Flores, R. y Anda, M. (1997). Dossier informativo sobre el tabaquismo y su prevención. Bilbao: Edex Kolektiboa.

Melero, J., Flores, R. y Anda, M. (1997). Cuadernos de actividades para el profesorado. Bilbao: Edex Kolektiboa.

Mendoza, R. (1999). Prevención del tabaquismo: un reto alcanzable. En Precioso, J., Viseu, F., Dourado, L., Vilaça, T, Henriques, R e Lacerda, T. (Coord.) (1999). Educação para a Saúde. Braga: Departamento de Metodologias da Educação. Universidade do Minho.

National Clearinghouse on Tobacco and Health (1993). Youth and tobacco: an adolescent health problem. Ontario: Canadian Council on Smoking and Health.

Nebot, M. (1999). Prevención del tabaquismo en los jóvenes. En Precioso, J., Viseu, F., Dourado, L., Vilaça, T, Henriques, R e Lacerda, T. (Coord.) (1999). Educação para a Saúde. Braga: Departamento de Metodologias da Educação. Universidade do Minho.

Nunes, E. (2002). Consumo de tabaco: Estratégias de Prevenção e Controlo. Lisboa: Cadernos da Direcção Geral de Saúde

Nutbeam, D., Mendoza, R. y Newman, R. (1988). Planning for a smoke - free generation. Copenhague: Regional Office for Europe of the World Health Organization.

Pestana, E. y Mendes, B. (1999). Tabagismo: 25 perguntas frequentes em pneumologia. Lisboa: Sociedade Portuguesa de Pneumologia.

Perry, C. y Forster, J. (2002). Youth Smoking: Can it Be Prevented or Reduced? TC online presetatons. Tobacco Control. www.tobaccocontrol.com.

Precioso, J. (2000). "Não fumar é o que está a dar: guia para o professor". Braga: Casa do professor. 
Precioso, J. (2001). "Aprende a cuidar de ti". Braga: Associação para a Prevenção e Tratamento do Tabagismo de Braga.

Precioso, J. (2003). A necessidadade de Prevenir e Promover o abandono do Tabagismo em Estudantes Universitários (en prensa).

Ribeiro, S. (1988). O Tabaco, a criança e o adolescente. Projecto "Tabaco ou Saúde"-relatório de actividades de 1987/88, (no publicado), Lisboa: Movimento Tabaco ou Saúde-Escolha Saúde.

Sanz, C. (1993). Tabaco, Alcohol y Educacion: una actuacion preventiva. Toledo: Comunidad de Castilla- La Mancha

Sanz, C. y Gándara, V. (1997). Efectividad de tres intervenciones para la prevención del consumo de tabaco en medio escolar. Revista de Psicología General y Aplica$d a, 50$ (2), 223-241.

Savolainen, S. (Ed.) (1998). The Smokefree class competition: a european school- based anti-smoking campaign; an overview of the 1997/78 round in seven countries. Helsinki: European of Network and Young People and
Tobacco. National Public Health Institute. Department of Epidemiology and Health Promotion.

U.S.D.H.H.S. (U.S. Department of Health and Human Services) (1994a). Preventing Tobacco Use Among Young People: A Report of the Surgeon General. Atlanta: Public Health Services. Centers for Disease Control and Prevention.

Ulster Cancer Foundation (1988). Smoking: basic facts. Belfast: Tacade/ASH.

Vries, H. (1989). Smoking prevention in Dutch adolescents. Maastricht: Dutch Cancer Foundation.

Vries, H. y Kok, G. (1986). From determinants of smoking behavior to the implications for a prevention programme. Health Education Research, 1 (2), 85-94

Vries, H., Dijkstra M. y Kuhlman, P.. (1988). Self-efficacy: the third factor besides attitude and subjective norm as a predictor of behavioral intentions. Health Education Research, 3, 273-282

Wiborg, G. y Hanewinkel, R. (1999). Smokefree Class Competition: A Scholl-Based Prevention Programme in Europe. Interaction, 12, 15-16. 
